initiation of treatment 'in the field', the need for administration by skilled personnel and risk of serious hemorrhagic complications. At present less than $5 \%$ of stroke patients are treated with thrombolysis. Neuroprotective interventions aim to limit neuronal damage. If successful stroke neuroprotective interventions are ultimately developed and if particularly these can be administered by EMS personnel at the time of initial interaction with the stroke victim, these would potentially extend the therapeutic window for thrombolysis as well as improving ultimate recovery. Neuroprotective strategies would likely be utilized empirically, sometimes in combination with thrombolysis when indicated.

Numerous trials of potentially 'neuroprotective' agents to date have failed to demonstrate a neuroprotective benefit in stroke patients despite initially promising outcomes in stroke models. The failure of anti-excitotoxic strategies in clinical stroke trials has necessitated a re-evaluation of excititotoxic mechanisms as the major mediator of cellular damage following stroke, as well as leading to an exploration of alternative hypotheses.

"New Strategies in Stroke Intervention" provides a succinct review of several novel concepts which might prove to be of ultimate clinical relevance in stroke management. The hardcovered book, comprising 250 pages of text with a four page index, is multi-authored, and comprises 12 chapters which provide specific overviews of a variety of channels, pumps and ion exchangers which might contribute to brain ischemic damage and which provide potential targets for therapeutic invention. Each chapter follows a similar format, providing details concerning the particular molecular entity under review including molecular structure, tissue distribution, biochemical and electrochemical properties, physiology and pathophysiological relevance to stroke, with further information concerning potential therapeutic pharmocomodulation and clinical trial data if available. Each chapter provides an index typically listing 50-150 references. The information provided will be understandable to clinicians who might not have extensive genetic, molecular or biophysiological or pharmacological backgrounds.

Initial chapters include an introductory review of ionic dysregulation in brain ischemia and a discussion of why current anti-excitotoxic interventions have failed. Subsequent chapters include discussions of mitochondrial channels, endoplasmic reticulum calcium homeostasis, $\mathrm{Na}^{+} / \mathrm{Ca}^{++}$exchanger, $\mathrm{Na}^{++} / \mathrm{H}^{+}$ exchanger, $\mathrm{Na}^{+} / \mathrm{K}^{+}$ATPase, acid-sensing ion channels, TRMP7 channels, voltage-gated $\mathrm{Ca}^{++}$channels and $\mathrm{K}^{+}$channels. The final chapter provides a tabulation of pharmaceutical trials targeting these various molecules including the status of these trials at the time of publication, treatment time from onset, and a brief commentary concerning outcome, adverse events and trial problems. The majority of chapters provide one or two figures, typically illustrating molecular structure or biochemical/path physiological pathways; figures are not indexed.

The publication will be of particular interest to Stroke clinicians and Fellows and to other Neuroscientists engaged in brain ischemia research.
The Mystery of Yawning in Physiology and Disease. Frontiers of Neurology and Neuroscience - Volume 28 Volume 28. 2010. Edited by Olivier Walusinski. Published by Basel, Karger. 159 pages. $C \$ 200$ approx.

\section{Rated UNAVAILABLE}

This book is Volume 28 in the series 'Frontiers of Neurology and Neuroscience' and it represents a summary of current knowledge about yawning, with contributors from eight countries under the editorship of a French primary care physician who himself contributes five sections including an historical survey.

It has long been accepted that yawning is a phylogenetically old behavior exhibited by humans, nonhuman primates and other mammals, birds and reptiles; that it is frequently contagious; and that it is especially associated with boredom. Yet, as the title of the book indicates, the function of yawning remains a mystery. In seeking an answer to this, data about its relationships and antecedents need to be reconciled. From various chapters, one learns that yawning is seen in fetuses after 12 weeks gestation and has been claimed to be associated with hunger, thirst, the need for physical love, boredom, low vigilance; thinking, witnessing or reading about yawning; awakening, recent fearfulness, cerebral hyperthermia (?where) and various disorders such as intracranial hypertension, after stroke, during opioid detoxification, as portents of seizures or migraine attacks, and in tic disorder and the Marin Amat syndrome, while it is uncommon in people with Parkinsonism or autism spectrum disorder and in castrated male rats (though testosterone restores that function to them.) Pontine, mesodiencephalic (mainly paraventricular nuclei) and prefrontal cortical disorders have all been incriminated in causation, and the behavior has been shown to require the activation of at least 11 neurotransmitters or neurohormones, though D3 receptor activation seems to be absolutely necessary. The occurrence of associated movements in hemiplegic limbs during a yawn demonstrates its dependence upon more than one motor pathway. Dr. Walusinski has coined the term 'parakinesia brachialis oscitans' for this phenomenon; only time will tell if this finds favour.

Opinions here seem to differ as to the roles of yawning as an erotic manifestation and in cortical arousal, but a consensus is that at least contagious yawning (discussed at length here in three chapters) is a primitive expression of social cognition (although I yawned repeatedly while reading the book in the sole company of my black Labrador, who yawned empathetically with me.)

This book will appeal to a limited readership, but it does nicely gather together many (but varied) opinions on the subject and will be absolutely invaluable to anyone starting inquiry in this field. The word for the scientific study of yawning is 'chasmology'. Ignoring the suggestive association of the words 'yawning' and 'chasm' in relation to our knowledge of the teleologic function of this activity, I have to say that I finished reading the book feeling like Dylan Thomas did after receiving from his aunt at Christmas one which told him everything he wanted to know about wasps - except why. 